\title{
Identificação anatômica de amostras comer- cializadas como espinheira-santa na Região Metropolitana do Rio de Janeiro
}

\section{Anatomical identification of samples sold as espinheira-santa in the metropolitan area of} Rio de Janeiro

Gabriel Uriel Cruz Araújo dos Santos $1^{\bullet \bullet}$, Eduarda Assis Freitas $1^{\bullet}$, Larissa Canutt Almeida Gomes $1^{\bullet} \bullet$, Marcio Junio de Azevedo Goudard $1^{\oplus} \bullet$, Felipe Gouvêa Guimarães $1^{\oplus} \bullet$, Beatriz da Rocha Gomes da Silva $1^{\bullet} \bullet$, Jeniffer Fonseca Go-

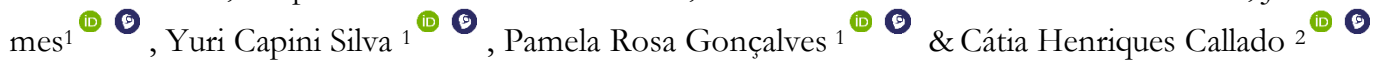

1. Colégio Pedro II, campus Duque de Caxias, Duque de Caxias, Rio de Janeiro, Brasil

2. Universidade do Estado do Rio de Janeiro, Instituto de Biologia Roberto Alcantara Gomes, Departamento de Biologia Vegetal, Laboratório de Anatomia Vegetal, Rio de Janeiro, Rio de Janeiro, Brasil

Palavras-chave:

Monteverdia ilicifolia. Sorocea bonplandii.

Plantas medicinais.

Keywords:

Monteverdia ilicifolia. Sorocea bonplandii.

Medicinal plants.

Recebido em: 24/03/2021

Aceite: 06/07/2021

Editor responsável: Gleidson V. Marques (UFSB)

eISSN: 2595-6752

(c) (i)

\section{Resumo}

Sabe-se que três espécies são comercializadas no Brasil sob o nome "espinheira-santa": Monteverdia ilicifolia (Mart. ex Reissek) Biral (Celastraceae), Sorocea bonplandii (Baill.) W.C.Burger, Lanj. \& Wess.Boer (Moraceae) e Zollernia ilicifolia Vogel (Leguminosae). Somente M. ilicifolia, no entanto, é reconhecida por suas propriedades medicinais, enquanto as demais são potencialmente tóxicas. Nosso objetivo foi verificar a identidade botânica das "espinheiras-santa" comercializadas em cidades da Região Metropolitana do RJ. Adquirimos 19 amostras oriundas principalmente de feiras, mercados municipais e lojas de produtos naturais. Para identificação do material, foram feitos cortes à mão livre do terço médio de folhas reidratadas, e as descrições anatômicas comparadas com a literatura e com lâminas de referência. Tais amostras frequentemente correspondem a uma fraude, já que apenas $1 \mathrm{em}$ cada 4 foram identificadas como M. ilicifolia. A anatomia vegetal é uma excelente ferramenta para o controle de fraudes em plantas medicinais devido ao seu baixo custo e simplicidade.

\section{Abstract}

In Brazil, three different species are currently sold as "espinheira-santa": Monteverdia ilicifolia (Mart. ex Reissek) Biral (Celastraceae), Sorocea bonplandii (Baill.) W.C.Burger, Lanj. \& Wess.Boer (Moraceae) and Zollernia ilicifolia Vogel (Leguminosae). However, only $\underline{M}$. ilicifolia is formally recognized for its medicinal properties, while the other two species are potentially toxic. Our aim was to verify the identity of the "espinheira-santa" sold in cities of the metropolitan region of Rio de Janeiro. We acquired 19 samples, mainly from fairs, city markets and natural products stores. In order to identify the material, we made free-hand anatomical sections of the midrib of rehydrated leaves, and the anatomical descriptions were compared to literature data and to reference slides. Only 5 out of 19 samples matched $\underline{M}$. ilicifolia, while the others mainly corresponded to $\underline{S}$. bonplandii. Plant anatomy is an excellent tool for fraud control in medicinal plants due to its low cost and simplicity.

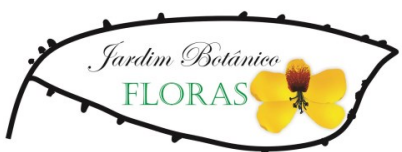




\section{Introdução}

A espinheira-santa, também conhecida como cancorosa e cancorosa-de-sete-espinhos (entre outros), é uma planta medicinal bastante utilizada na medicina popular brasileira (Coulaud-Cunha et al., 2004; Mariot; Barbieri, 2007a; Santos-Oliveira et al., 2009; Jesus; Cunha, 2012; Almeida et al, 2015; Martinelli et al., 2018; Périco et al., 2018). Suas folhas são utilizadas popularmente para tratamento de problemas estomacais no geral, dores no estômago, úlcera, gastrite, ingestão exagerada de alimentos, refluxo, azia, cicatrização e limpeza do sangue (Mariot; Barbieri 2007a; Almeida et al., 2015; Alcantara et al., 2015). Pesquisas apontam para suas propriedades farmacológicas, como antiulcerogênica, analgésica, anti-inflamatória, abortiva, cicatrizante, entre outras (Gonzales et al., 2001; Montanari; Bevilacqua, 2002; Jorge et al., 2004; Santos-Oliveira et al., 2009).

No Brasil, sob o nome popular "espinheira-santa", no entanto, são comercializadas três espécies de famílias diferentes: Monteverdia ilicifolia (Mart. ex Reissek) Biral (Celastraceae) (sinônimo: Maytenus ilicifolia Mart. ex Reissek - Biral et al., 2017; Flora do Brasil 2020), Sorocea bomplandii (Baill.) W.C.Burger, Lanj. \& Wess.Boer (Moraceae) e Zollernia ilicifolia Vogel (Leguminosae). Estas possuem o mesmo nome popular por apresentarem folhas morfologicamente semelhantes (Alberton et al., 2002; Machado; Santos, 2004).

A Farmacopeia Brasileira (ANVISA, 2019) reconhece a droga vegetal somente como folhas secas de $M$. ilicifolia, mas $S$. bonplandii $\mathrm{e}$ Z. ilicifolia são frequentemente utilizadas em seu lugar (Parente; Rosa, 2001; Alberton et al., 2002; Machado; Santos, 2004; CoulaudCunha et al., 2004; Coelho et al., 2007; Giraldi; Hanazaki, 2010; Jesus; Cunha, 2012; Bochner et al., 2012), tendo relatos também do uso de Sorocea guilleminiana como espinheira-santa (Maioli-Azevedo; Fonseca-Kruel, 2007). Apesar de algumas pesquisas mostrarem efeitos farmacológicos de S. bonplandii e Z. ilicifolia (por exemplo, Gonzales et al., 2001), seu uso desperta preocupação. Leitão et al. (2014) assinalam o risco da população utilizar-se de plantas tóxicas por conta da identificação errônea de espinheira-santa. CoulaudCunha et al. (2004) e Leitão et al. (2014) afirmam que faltam estudos que comprovem a eficácia e a ausência de toxicidade de $S$. bonplandii. Gonzales et al. (2001) mostraram sinais de toxicidade aguda com o uso de Z. ilicifolia, e Coelho et al. (2007) relatam a presença, nesta espécie, de componentes tóxicos que podem causar a morte. Rosa et al. (2013) também demonstraram ausência de atividade gastroprotetora com Z. ilicifolia. Desta forma, apenas o uso de M. ilicifolia pode ser considerado seguro, e as demais espécies sendo consideradas adulterantes.

A anatomia vegetal tem sido utilizada para a identificação de plantas medicinais comercializadas, especialmente quando compostas somente por material vegetativo. Engel et al. (2008) utilizaram a anatomia foliar para identificar espécies adulterantes de Baubinia forficata Link em Itajaí e Balneário Camboriú. Macrini (2011) utilizou análises microscópicas de diversas partes vegetais de nove espécies, como ginco (Ginkgo biloba L. - Ginkgoaceae), maracujá (Passiflora alata Curtis e P. edulis Sims - Passifloraceae), marapuama (Ptychopetalum olacoides Benth. - Olacaceae) e valeriana (Valeriana officinalis - Valerianaceae). Costa et al. (2014) identificaram adultera- ções em amostras de chá verde (Camelia sinensis (L.) Kuntze) e espinheira-santa adquiridas em estabelecimentos no Rio de Janeiro (RJ) e em Taubaté (SP). Miranda et al. (2016) encontraram adulterações em aroeira (Schinus terebinthifolius Raddi) vendida em mercados públicos de São Luís (MA) utilizando a anatomia da casca.

Tendo em vista a venda de espécies adulterantes de espinheira -santa, que podem oferecer risco à população, este trabalho objetivou analisar, através da micromorfologia, amostras à venda na Região Metropolitana do Rio de Janeiro, para identificar quais espécies estão sendo comercializadas.

\section{Material e Métodos}

As amostras analisadas consistem em material vegetal vendido sob o nome "espinheira-santa", em lojas de produtos naturais, feiras livres e mercados populares. Foram adquiridas 19 amostras em 5 municípios da Região Metropolitana do estado do Rio de Janeiro (Tabela 1, Figura 1).

As amostras foram reidratadas em laboratório, infundindo folhas em água e aquecendo em micro-ondas até atingir fervura e as folhas submergirem por completo. Das folhas reidratadas foram selecionados fragmentos de aproximadamente $1 \mathrm{~cm}^{2}$ no terço médio, ao redor da nervura central. As amostras foram secionadas à mão livre e coradas com solução de Azul de Astra e Safranina (Bukatsch, 1972). Lâminas semipermanentes foram montadas e analisadas ao microscópio ótico Olympus BX 41 e imagens foram obtidas com auxílio da câmera Q Collor 5 .

As amostras foram descritas e comparadas tanto com material de referência (lâminas de Monteverdia ilicifolia da Unidade de Desenvolvimento Tecnológico Laboratório de Anatomia Vegetal da Universidade do Estado do Rio de Janeiro) quanto com a literatura disponível, tanto para M. ilicifolia e outras espécies de Monteverdia A. Rich., quanto para Sorocea bonplandii (Baill.) W.C.Burger, Lanj. \& Wess.Boer e Zollernia ilicifolia Vogel (Jacomasi; Machado, 2003; Machado; Santos, 2004; Rocha et al., 2004; Joffily; Vieira, 2005; Duarte; Debur, 2005) para fins de identificação.

Após identificadas, as amostras foram contadas e foi feito um gráfico no Microsoft Excel versão $16.0 \mathrm{com}$ as proporções de amostras identificadas corretamente e das amostras identificadas erroneamente.

\section{Resultados e Discussão}

As amostras 4, 7, 10, 14 e 17 (Tabela 1) apresentaram estrutura anatômica muito semelhante, caracterizadas por nervura central com face adaxial convexa acentuada (Figura 2a), face abaxial convexa atenuada, colênquima anelar reduzido, anel de fibras perivasculares contínuo e anel de floema contínuo (Figura 2c). Essas características são atribuídas a $M$. ilicifolia e ausentes em S. bomplandii. (Jacomasi; Machado, 2003; Machado; Santos, 2004). Cristais do tipo aciculares ou ráfides na epiderme da lâmina foliar são comuns em $M$. ilicifolia (Jacomasi; Machado, 2003; Machado; Santos, 2004) e em outras espécies do gênero Monteverdia (Joffily; Vieira, 2005), mas não foram observados nessas amostras. Estratos subepidérmicos aclorofilados, 
Figura 1: Localização dos pontos de coleta das amostras de espinheira-santa. Modificados a partir de IBGE (mapa do estado do RJ) e Prefeitura da cidade do Rio de Janeiro (Região Metropolitana do RJ).
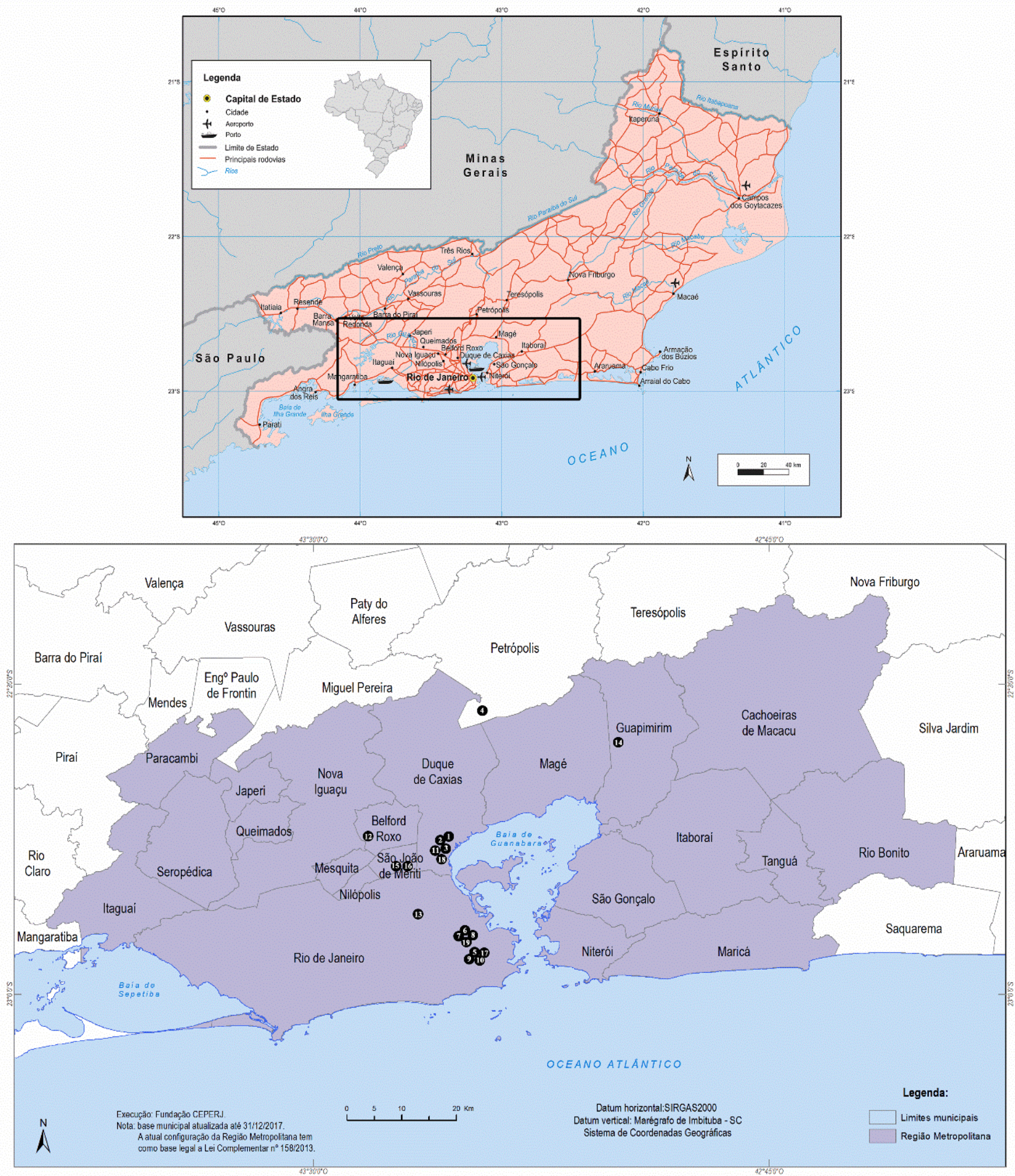
Figura 2: Seções transversais do terço médio de amostras de espinheira-santa. a. Monteverdia ilicifolia. Face adaxial (Ad) em formato convexo acentuado e face abaxial (Ab) em formato convexo atenuado. b. Sorocea bonplandii. Face adaxial (Ad) em formato côncavo e face abaxial (Ab) em formato convexo acentuado. c. Anel de fibras perivasculares contínuo (seta branca), colênquima (Col) reduzido na face abaxial. d. Anel de fibras perivasculares e floema interrompidos por parênquima (setas), colênquima $(\mathrm{Col})$ amplo na face abaxial. Barra $=250 \mu \mathrm{m}$ em a e b e $100 \mu \mathrm{m}$ em c e d.
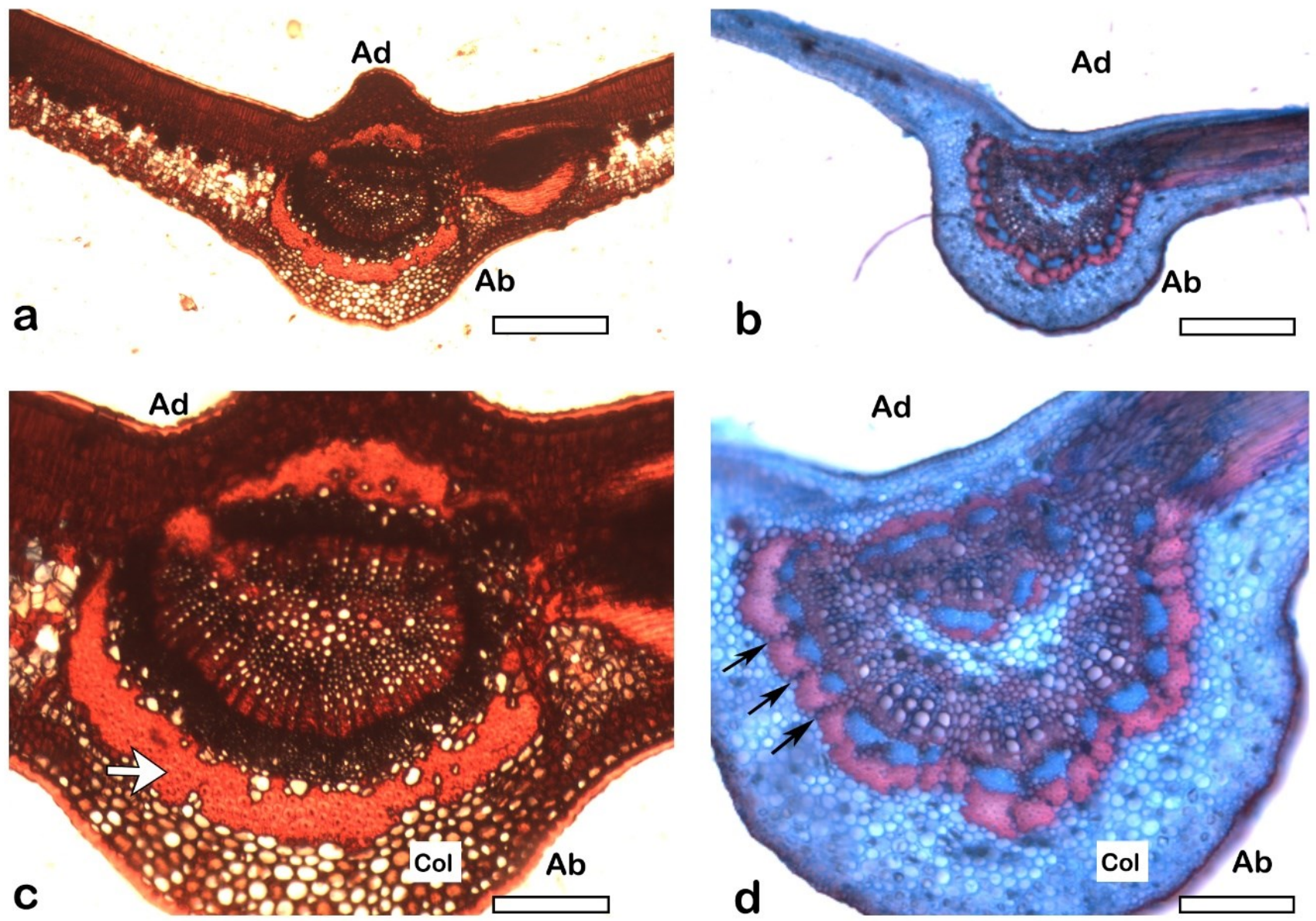

Figura 3: Quantidade de amostras identificadas corretamente como Monteverdia ilicifolia e de amostras identificadas como outras espécies.

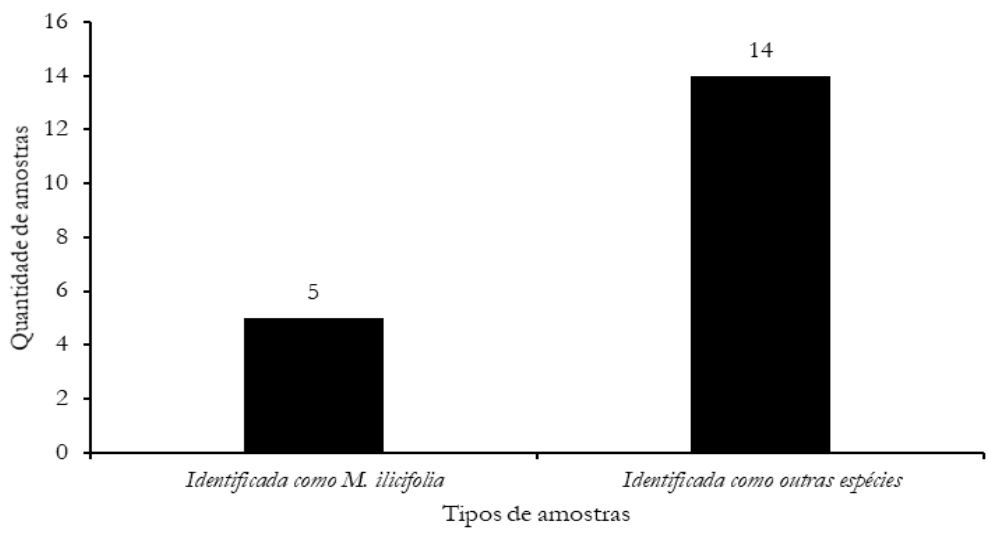


Tabela 1. Amostras de espinheira-santa adquiridas na Região Metropolitana do estado do RJ.

\begin{tabular}{|c|c|c|}
\hline Amostra & Local (bairro, cidade) & Tipo de estabelecimento \\
\hline 1 & Centro, Duque de Caxias & Mercado municipal \\
\hline 2 & Centro, Duque de Caxias & Mercado municipal \\
\hline 3 & Centro, Duque de Caxias & Mercado municipal \\
\hline 4 & Bairro não conhecido, Petrópolis & Sítio \\
\hline 5 & Tijuca, Rio de Janeiro & Feira livre \\
\hline 6 & Vila Isabel, Rio de Janeiro & Feira livre \\
\hline 7 & Vila Isabel, Rio de Janeiro & Loja de produtos naturais \\
\hline 8 & Vila Isabel, Rio de Janeiro & Banca de jornal \\
\hline 9 & Tijuca, Rio de Janeiro & Banca de jornal \\
\hline 10 & Tijuca, Rio de Janeiro & Loja de produtos naturais \\
\hline 11 & Centro, Duque de Caxias & Loja de produtos naturais \\
\hline 13 & Madureira, Rio de Janeiro & Mercado municipal \\
\hline 14 & Paiol, Guapimirim & Loja de produtos naturais \\
\hline 15 & Vilar dos Teles, São João de Meriti & Feira livre \\
\hline 16 & Vilar dos Teles, São João de Meriti & Feira livre \\
\hline 17 & Tijuca, Rio de Janeiro & Loja de produtos naturais \\
\hline 18 & Centro, Duque de Caxias & Loja de produtos naturais \\
\hline 19 & Vila Isabel, Rio de Janeiro & Loja de produtos naturais \\
\hline
\end{tabular}

ausentes dessas amostras, ocorrem em M. obtusifolia (Joffily; Vieira, 2005) e em Zollernia ilicifolia (Jacomasi; Machado, 2003; Machado; Santos, 2004). Z. ilicifolia também é caracterizada pela presença de lacticíferos na nervura central (Jacomasi; Machado, 2003; Machado; Santos, 2004), ausentes aqui. Essas amostras são muito semelhantes às lâminas de referência da UERJ. Portanto, conclui-se que se tratam de amostras de Monteverdia, provavelmente M. ilicifolia.

As amostras 1, 2, 3, 5, 6, 8, 9, 11, 12, 13, 15, 18 e 19 (Tabela 1) são anatomicamente muito semelhantes entre si. São caracterizadas por nervura central com face adaxial côncava, face abaxial convexa acentuada (Figura 2b), colênquima anelar abundante, cristais (grandes prismas, ocasionalmente drusas) localizados nos tecidos ao redor da nervura, anel de fibras perivasculares interrompido por parênquima e floema em pequenos grupos separados por parênquima (Figura 2d) . Combinados com a ausência de lacticíferos na nervura central, ráfides na epiderme da lâmina foliar e estrato subepidérmico aclorofilado na lâmina foliar (Jacomasi; Machado, 2003; Machado; Santos, 2004), conclui-se que se tratam de amostras do gênero Sorocea A. St.-Hil., provavelmente $S$. bonplandii, com base nas descrições de Marques et al. (1976).

A amostra 16 foi macroscopicamente identificada como Baubinia sp., conhecida popularmente como pata-de-vaca. Como a morfologia das plantas deste gênero difere em muito das plantas comercializadas como espinheira-santa (ANVISA, 2019), consideramos esta adulteração como bastante séria.

$\mathrm{Na}$ nossa amostragem, as identificações corretas de espinheira -santa representam aproximadamente $26 \%$ (5 de 19), enquanto as identificações errôneas representam aproximadamente 74\% (14 de 19) (Figura 2). A prevalência de amostras de S. bonplandii não surpreende, visto que já foi constatado anteriormente a abundante venda desta espécie como adulterante: Coulaud-Cunha et al. (2004) analisaram amostras vendidas em feiras livres na cidade do Rio de Janeiro e afirmam que a maioria deles obtinham a planta do mesmo produtor (CEASA), o que justificaria a amplitude da adulteração. Isso corrobora os nossos resultados, visto que as amostras da verdadeira espinheira-santa foram quase todas obtidas em lojas de produtos naturais (Tabela 1 ).

Entretanto, é digno de nota que a amostra 19 foi uma repetição da amostragem do mesmo local em que foi obtida a amostra 7. A primeira foi identificada como $S$. bonplandii, e a última como M. ilicifolia. O local de compra é uma loja de produtos naturais, e as amostras foram obtidas com alguns meses de diferença. Desta forma, consideramos que a fiscalização da venda de plantas medicinais deve ser constante, a fim de garantir o controle de qualidade das amostras.

Nossos resultados corroboram a importância do uso da anatomia vegetal na identificação de plantas medicinais comercializadas. Muitas vezes o material vendido não pode ser identificado macroscopicamente, devido à fragmentação do mesmo e à ausência de partes reprodutivas. Diversos autores, como Engel et al. (2008), Macrini (2011), Costa et al. (2014) e Miranda et al. (2016), foram capazes de confirmar ou afastar adulterações, em diversas espécies medicinais, utilizando a anatomia de partes vegetativas comercializadas.

\section{Conclusão}

Os produtos comercializados nos locais amostrados frequentemente correspondem a uma fraude, já que apenas 1 em cada 4 amostras foram identificadas como M. ilicifolia. Concluímos, também, que o controle de adulterações através da anatomia vegetal é relativamente simples e de baixo custo, o que a torna uma excelente ferramenta para o controle de fraudes de plantas medicinais. 


\section{Agradecimentos}

Os autores agradecem imensamente à Mariana Cabral, pelo apoio técnico ao projeto no campus Duque de Caxias; aos integrantes do Laboratório de Anatomia Vegetal, especialmente a Jeanne Teixeira, Thaís Jorge e Daiane Silva, pelo apoio técnico nos cortes, nas fotos e nas pranchas; aos professores do Colégio Pedro II, Elizabeth Pasin, Paula Mello, Vanessa Gonçalves, Natasha Carvalho, Leandro Seixas e Christiane Coelho, pelo apoio ao projeto de Iniciação Científica que resultou neste artigo.

Financiamento

Parte dos autores (Beatriz da Rocha Gomes Silva, Larissa Canutt Almeida Gomes, Marcio Junio de Azevedo Goudard e Felipe Gouvêia Guimarães) recebeu bolsa de Iniciação Científica da Pró-reitoria de Pós-Graduação, Pesquisa, Extensão e Cultura (PROPGPEC) do Colégio Pedro II.

Contribuições de Autoria

Conceitualização: GUCAS. Administração do projeto: GUCAS, PRG. Aquisição de financiamento: GUCAS, PRG, CHC. Investigação: GUCAS, PRG, BRGS, LCAG, MJAG, FGG, JFG, YCS, EAF, CHC. Redação - rascunho original: GUCAS. Redação revisão e edição: CHC, PRG, BRGS, LCAG, MJAG, FGG, JFG, YCS, EAF.

\section{Conflito de Interesse}

Os autores declaram não haver conflitos de interesse a informar.

Disponibilidade dos Dados

Os dados integrais analisados durante o estudo atual podem ser fornecidos mediante solicitação justificada à autora para correspondência.

Conformidade ética

Não se aplica.

\section{Referências}

Alberton MD, Falkenberg DB, Falkenberg MB. Análise cromatográfica de fitoterápicos a base de espinheira-santa (Maytenus ilicifolia). Revista Brasileira de Famacognosia 2002;12(supl.):11-13. doi: 10.1590/S0102-695X2002000300006

Alcantara RGL, Joaquim RHVT, Sampaio SF. Plantas medicinais: o conhecimento e uso popular. Revista de APS 2015;18(4):470482.

Almeida C, Barbieri RL, Ribero MV, Lopes CV, Heck RM. Espinheira-santa (Maytenus ilicifolia Mart. ex Reiss.): saber de erveiros e feirantes em Pelotas (RS). Revista Brasileira de Plantas Medicinais 2015;17(4):722-729. doi: 10.1590/1983-084X/14_003

ANVISA. Farmacopeia brasileira. Vol. II - Monografias. Plantas Medicinais. Brasília; 2019.

Azevedo SKS, Silva IM. Plantas medicinais e de uso religioso comercializadas em mercados e feiras livres no Rio de Janeiro, RJ, Brasil. Acta Botanica Brasilica 2006;20(1):185-194. doi: 10.1590/ S0102-33062006000100017

Biral L, Simmons MP, Smidt EC, Tembrock LR, Bolson M, Archer RH, Lombardi JA. Systematics of New World Maytenus (Celastraceae) and a new delimitation of the genus. Systematic Botany 2017;42(4):680-696. doi: 10.1600/036364417X696456
Bochner R, Fiszon JT, Assis MA, Avelar KES. Problemas associados ao uso de plantas medicinais comercializadas no Mercadão de Madureira, município do Rio de Janeiro, Brasil. Revista Brasileira de Plantas Medicinais 2012;14(3):537-547. doi: 10.1590/ S1516-05722012000300017

Bukatsch F. Bemerkungen zur Doppelfärbung: Astrablau-Safranin. Mikrokosmos 1972;61: 255.

Chimin A, Lima EL, Beltrame FL, Pereira AV, Esmerino LA. Avaliação da qualidade de amostras comerciais de Maytenus ilicifolia (espinheira-santa) comercializadas no Estado do Paraná. Latin American Journal of Pharmacy 2008;27(4):591-597.

Coelho RG, Calvo TR, Santos LC, Di Stasi LC, Vilegas W. Separation of the toxic zierin from Zollernia ilicifolia by high speed countercurrent chromatography. Revista Brasileira de Plantas Medicinais 2007;9(4):39-43.

Costa RPC, Guimarães ALC, Vieira ACM. Avaliação da qualidade de amostras de plantas medicinais comercializadas no Brasil. Revista de Ciências Farmacêuticas Básica e Aplicada 2014;35 (3):425-433.

Coulaud-Cunha S, Oliveira RS, Waissmann, W. Venda livre de Sorocea bomplandii Bailon como Espinheira Santa no município de Rio de Janeiro- RJ. Revista Brasileira de Farmacognosia 2004;14 (supl. 1):51-53. Doi: 10.1590/S0102-695X2004000300019

Duarte MR, Debur MC. Stem and leaf morphoanatomy of Maytenus ilicifolia. Fitoterapia 2005;76:41-49. doi: 10.1016/ j.fitote.2004.10.003

Engel IC, Ferreira RA, Cechinel-Filho V, Meyre-Silva C. Controle de qualidade de drogas vegetais a base de Baubinia forficata Link (Fabaceae). Revista Brasileira de Farmacognosia 2008;18(2): 258264. doi: https://doi.org/10.1590/S0102-695X2008000200021

Flora do Brasil 2020 [Internet]. Rio de Janeiro: JBRJ [citado em 26 mai 2020]. Disponível em: http:// floradobrasil.jbrj.gov.br/

Giraldi M, Hanazaki N. Uso e conhecimento tradicional de plantas medicinais no Sertão do Ribeirão, Florianópolis, SC, Brasil. Acta Botanica Brasilica 2010;24(2):395-406. doi: 10.1590/S010233062010000200010

Gonzales FG, Portela TY, Stipp EJ, Di Stasi LC. Antiulcerogenic and analgesic effects of Maytenus aquifolium, Sorocea bomplandii and Zolernia ilicifolia. Journal of Ethnopharmacology 2001;77:41-47. doi: 10.1016/S0378-8741(01)00268-9

Jacomasi E, Machado SR. Características anatômicas de espinheirasanta (Maytenus ilicifolia Mart. ex Reissek e Maytenus aquifolia Mart.) e mata-olho (Sorocea bonplandii (Baill.) Burg. Lanj. \& Boer) para o controle de qualidade de matéria prima. Revista Brasileira de Plantas Medicinais 2003;6(1):84-93.

Jesus WMM, Cunha TN. Estudo das propriedades farmacológicas da espinheira santa (Maytenus ilicifolia Mart. ex Reissek) e de duas espécies adulterantes. Revista Saúde e Desenvolvimento 2012;2 (1):20-46.

Joffily A, Vieira RC. Anatomia foliar de Maytenus Mol. emend Mol. (Celastraceae), ocorrente no Estado do Rio de Janeiro, Brasil. Acta Botanica Brasilica 2005;19(3):549-561. Doi: 10.1590/S0102 $-33062005000300016$

Jorge RM, Leite JPV, Oliveira AB, Tagliati CA. Evaluation of antinociceptive, anti-inflammatory and antiulcerogenic activities of Maytenus ilicifolia. Journal of Ethnopharmacology 2004;94:93-100. Doi: 10.1016/j.jep.2004.04.019

Leitão F, Leitão SG, Fonseca-Kruel VS, Silva IM, Martins K. Medicinal plants traded in the open-air markets in the State of Rio de Janeiro, Brazil: an overview on their botanical diversity and toxicological potential. Revista Brasileira de Farmagnosia 2014;24:225-247. Doi: doi.org/10.1016/j.bjp.2014.04.005

Machado AV, Santos M. Morfo-anatomia foliar comparativa de espécies conhecidas como espinheira-santa: Maytenus ilicifolia 
(Celastraceae), Sorocea bonplandii (Moraceae) e Zollernia ilicifolia (Leguminosae). Insula 2004;33:1-19.

Macrini T. Análise farmacognóstica de amostras de drogas vegetais psicoativas comercializadas em Diadema [dissertação]. São Paulo, SP: Universidade de São Paulo; 2011.

Maioli-Azevedo V, Fonseca-Kruel VS. Plantas medicinais e ritualísticas vendidas em feiras livres no município do Rio de Janeiro, RJ, Brasil: estudo de caso nas zonas Norte e Sul. Acta Botanica Brasilica 2007;21(2):263-275. Doi: 10.1590/S010233062007000200002

Mariot MP, Barbieri RL. O conhecimento popular associado ao uso da espinheira-santa (Maytenus ilicifolia e M. aquifolium). Revista Brasileira de Biociências 2007a;5(supl. 1):666-668.

Mariot MP, Barbieri RL. Metabólitos secundários e propriedades medicinais da espinheira-santa (Maytenus ilicifolia Mart. ex Reiss. e M. aquifolium Mart.). Revista Brasileira de Plantas Medicinais 2007b;9(3):89-99.

Marques MC, Carauta JPP, Costa CG, Sucre D. O gênero Sorocea A. Saint Hilaire (Moraceae) no Estado do Rio de Janeiro: Anatomia e Taxonomia. Annaes da Academia Brasileira de Ciências 1976;48(2):285-300

Martinelli S, Oliveira AS, Santos RO, Winter MA, Dolce AV, Gheller ACGV. Controle de qualidade da droga vegetal "espinheirasanta" encontrada em mercados de Sinop/MT. FACIDER Revista Científica 2018;11:1-12.

Miranda MV, Firmo WCA, Pereira LPLA, Dias CN, Castro NG, Olea RSG, Moraes DFC, Silveira LMS. Controle de qualidade de amostras comerciais de Schinus terebinthifolius Raddi (Aroeira) adquiridas em mercados públicos da cidade de São Luís-MA. Biota Amazônia 2016;6(1):83-90. Doi: 10.18561/2179-5746/ biotaamazonia.v6n1p83-90

Montanari T, Bevilacqua E. Effect of Maytenus ilicifolia Mart. on pregnant mice. Contraception 2002;65:171-175. doi: 10.1016/ s0010-7824(01)00301-8

Nascimento VT, Lacerda EU, Melo JG, Lima CSA, Amorim ELC, Albuquerque UP. Controle de qualidade de produtos à base de plantas medicinais comercializados na cidade do Recife-PE: ervadoce (Pimpinella anisum L.), quebra-pedra (Phyllanthus spp.), espinheira santa (Maytenus ilicifolia Mart.) e camomila (Matricaria recutita L.). Revista Brasileira de Plantas Medicinais 2005;7(3):56-64.

Parente CET, Rosa MMT. Plantas comercializadas como medicinais no município de Barra do Piraí, RJ. Rodriguésia 2001;52:4759. doi: 10.1590/2175-78602001528004

Périco LL, Rodrigues VP, de Almeida LFR, Fortuna-Perez AP, Vilegas W, Hiruma-Lima CA. Maytenus ilicifolia Mart. ex Reissek. In: Albuquerque U, Patil U, Máthé Á. Medicinal and Aromatic Plants of South America. Medicinal and Aromatic Plants of the World, vol 5. Dordrecht: Springer; 2018. doi: 10.1007/978-94024-1552-0

Rocha CS, Pimentel RMM, Randau KP, Xavier HS. Morfoanatomia de folhas de Maytenus rigida Mart. (Celastraceae); uma espécie utilizada como medicinal no Nordeste do Brasil. Acta Farmacologica Bonaerense 2004;23(4):472-476.

Rosa T, Scaini G, Rezin GT, Maggi DD, Pezente DP, Valerio WL, Teodorak BP, Rossato AE, Amaral PA, Citadini-Zanette V, Streck EL. Avaliação do efeito do extrato hidroalcoólico de Zollernia ilicifolia (Fabaceae) como gastroprotetor em ratos. Revista Inova Saúde 2013; 2(2):74-89.

Santos-Oliveira R, Coulaud-Cunha S, Colaço W. Revisão da Maytenus ilicifolia Mart. ex Reissek, Celastraceae. Contribuição ao estudo das propriedades farmacológicas. Revista Brasileira de Farmacognosia 2009;19(2B):650-659. doi: 10.1590/S0102-

695X2009000400025

\section{Como citar este artigo \\ How to cite this article}

(ABNT)

SANTOS, G. U. C. A.; FREITAS, E. A.; GOMES, L. C. A.; GOU-

DARD, M. J. A.; GUIMARÃES, F. G.; SILVA, B. R. G.; GOMES, J. F.; SILVA, Y. C.; GONÇALVES, P. R.; CALLADO, C. H. Identificação anatômica de amostras comercializadas como espinheirasanta na Região Metropolitana do Rio de Janeiro. Paubrasilia, Porto Seguro, v. 4, e0064, 2021. DOI 10.33447/

paubrasilia.2021.e006

(Vancouver)

Santos GUCA, Freitas EA, Gomes LCA, Goudard MJA, Guimarães FG, Silva BRG, Gomes JF, Silva YC, Gonçalves PR, Callado CH. Identificação anatômica de amostras comercializadas como espinheira-santa na Região Metropolitana do Rio de Janeiro. Paubrasilia 2021;4:e0064. doi:10.33447/paubrasilia.2021.e006 